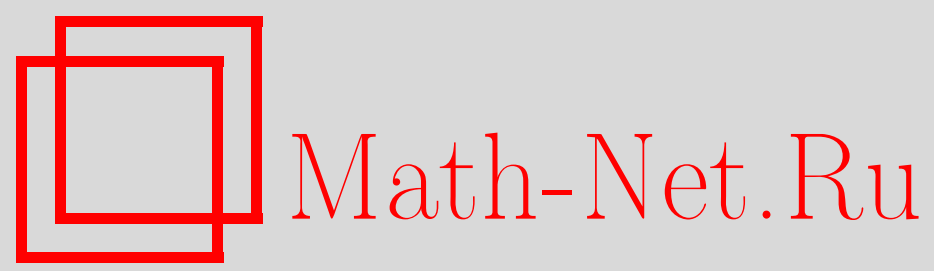

С. В. Ульянов, А. А. Мамаева, А. В. Шевченко, Технология когнитивного интеллектуального управления: оптимизатор баз знаний на мягких вычислениях и глубокое машинное обучение, Нечеткие системы и мягкие вычисления, 2018, том 13, выпуск 2, 166-182

DOI: https://doi.org/10.26456/fssc44

Использование Общероссийского математического портала Math-Net.Ru подразумевает, что вы прочитали и согласны с пользовательским соглашением

http: //www.mathnet.ru/rus/agreement

Параметры загрузки:

IP : 54.80 .97 .219

26 апреля 2023 г., 09:17:38 
УДК $510.676,519.7$

\section{ТЕХНОЛОГИЯ КОГНИТИВНОГО ИНТЕЛЛЕКТУАЛЬНОГО УПРАВЛЕНИЯ: ОПТИМИЗАТОР БАЗ ЗНАНИЙ НА МЯГКИХ ВЫЧИСЛЕНИЯХ И ГЛУБОКОЕ МАШИННОЕ ОБУЧЕНИЕ}

Ульянов С.В., Мамаева А.А., Шевченко А.В.

Государственный университет «Дубна», г. Дубна

Поступила в редакцию 05.12.2018, после переработки 17.12.2018.

В статье обсуждаются возможности использования технологии глубокого машинного обучения на основе оптимизатора баз знаний на мягких вычислениях в задачах когнитивного интеллектуального управления с применением когнитивного шлема в качестве нейроинтерфейса. Цель данной работы: экспериментально показать возможность классификации ментальных состояний человека-оператора, выявить объективные показатели психофизиологического состояния исследуемого лица с применением технологий мягких вычислений. Показана на примерах роль и необходимость применения интеллектуальных вычислений в задаче описания общего психофизического состояния оператора.

Ключевые слова: нейроинтерфейс, интеллектуальные вычисления, интеллектуальная система управления, глубокое машинное обучение, эмоции.

Нечеткие системы и мягкие вычисления. 2018. Т. 13, № 2. С. 166-182. https://doi.org/10.26456/fssc44

\section{1. Введение: проблемы и задачи когнитивного интеллектуального управления}

Включение человека-оператора в интеллектуальные системы управления (ИСУ) для принятия решения в сложных ситуациях создает информационный ресурс, который позволяет повысить эффективность разработки и применения ИСУ. Однако он часто связан с повышением риска ситуаций из-за наличия непредсказуемого человеческого фактора [1]. Именно поэтому необходимо иметь количественные и качественные показатели, которые бы не зависели от индивидуальньх характеристик человека-оператора, и при этом гарантировалась, в определенном смысле, объективность полученных показателей. Тогда разрабатываемые ИСУ будут способны к восприятию, адаптации и принятию решений в сложных ситуациях [2] за счет включения в структуру данных показателей как критериев качества интеллектуального управления.

Ряд исследований $[3,4]$ показал возможность построения упрощенных математической модели эмоций. Но, помимо физических ограничений, на корректность описания и достоверность извлеченных знаний из математической модели существенное влияние оказывают также и информационные границы на применимость разработанной модели. В теории ИСУ одним из эффективных подходов 
снижения риска принятия решения (от неполноты описания модели ОУ) является разработка робастных структур ИСУ. Проблемы физических ограничений и информационных границ решаются возможностью формирования БЗ с требуемым уровнем робастности в процессе проектирования ИСУ путем извлечения знаний и ценной информации из динамического поведения модели самого физического OУ [5].

Однако, структуры ИСУ не обладают специализированным программным модулем для описания и реализации процессов адаптации и обучения системы управления к качественным особенностям поведенческих реакций человекаоператора.

В свою очередь, зарекомендовавшие себя в широком классе областей мягкие вычисления (генетические алгоритмы, нечеткая логика и нечеткие нейронные сети) и технология интеллектуальных вычисления в виде интеллектуального инструментария (Computational Intelligence Toolkit), позволяют спроектировать интеллектуальную когнитивную систему управления, обладающую требуемыми качествами.

Цель данной работы: экспериментально показать возможность классификации ментальных состояний человека-оператора, выявить объективные показатели психофизиологического состояния исследуемого лица на основе глубокого машинного обучения с применением интеллектуального инструментария в виде оптимизатора баз знаний на мягких вычислениях.

\section{2. Снятие и обработка ЭЭГ}

Электроэнцефалограмму (ЭЭГ) человека-оператора можно использовать в качестве биометрического параметра, поскольку мозговая активность индивидуальна. Уникальным ее делает синхронизированная активность групп нейронов. Нейроны, обрабатывающие одни и те же сигналы, формируют метастабильные группы. Сигналы, соответствующие одному внешнему стимулу или когнитивному событию, вызывают синхронизированную активность объединившихся в группы нейронов. Определенный уровень такой синхронизации сохраняется и в состоянии покоя. Синхронизированная активность нейронов наблюдается на ЭЭГ.

\section{3. Особенности экспериментальной регистрации ЭЭГ и информатив- ные параметры состояния пациента}

Снятие сигнала ЭЭГ - контактная и продолжительная процедура, поскольку электрическая активность мозга - величина, протяженная во времени, при этом данные нельзя снимать длительное время - на больших промежутках времени проявляются нелинейные искажения сигнала ЭЭГ. Нелинейность сигнала решается серией коротких замеров, в течении которых сигнал можно считать линейным.

Для снятия сигнала о мозговой активности использовался когнитивный шлем Emotiv EPOC+ (см. Рис. 1 и 2).

ЕРОС имеет 14 электродов, которые являются пассивными сенсорами, позволяющими регистрировать электромагнитные сигналы. Датчики крепятся на поверхности кожи (не погружной, неинвазивный интерфейс). На Рис. 2 представлена структура Emotiv EPOC+, состоящего из каналов AF3, F7, F3, FC5, T7, P7, 


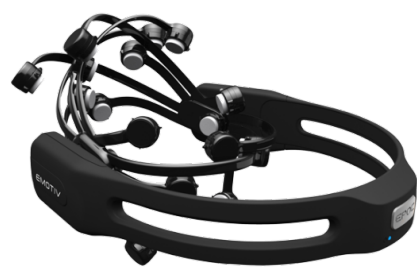

Puc. 1: Когнитивный шлем Emotiv $\mathrm{EPOC+}$

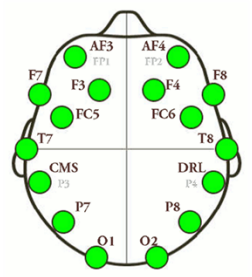

Рис. 2: Схема расположения электродов когнитивного илема Emotiv EPOC+

O1, O2, P8, T8, FC6, F4, F8, AF4 и двух референсных датчиков CMS/DRL, задача которых получать и отфильтровывать от сигнала ЭЭГ биоэлектрические сигналы мышечной активности. Поставляемое программное обеспечение позволяет получать, распознавать и регистрировать ЭЭГ сигнал со шлема [6]. В рамках решения задачи необходимо получить наиболее информативные фрагменты сигнала. Для анализа выделяются частотные ритмы ЭЭГ. Понятие частотный ритм определяет тип электрической активности, соответствующий некоторому состоянию мозга, для которого определены границы диапазона частот (см. Рис. 3).

\section{Beta}

[12-30 Hz]
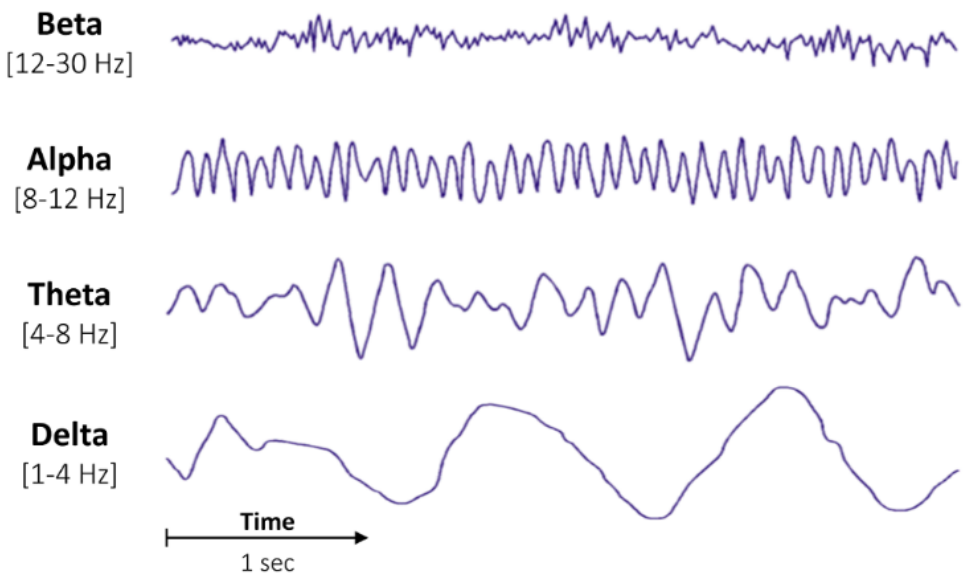

Рис. 3: Частотные ритмы ЭЭГ

Это подразумевает разложение сигнала ЭЭГ на частотные составляющие, что достигается посредством быстрых преобразований Фурье (БПФ), которое возвращает для каждого частотного буфера комплексное число, содержащее амплитуду и фазу:

$$
X_{k}=\sum_{n=0}^{N-1} x_{n} \mathrm{e}^{-i 2 \pi k n / N},
$$

где $N$ - количество временных выборок, $k=0, \ldots, N-1$ - текущая частота, $n=0, \ldots, N-1$ - текущая выборка. $x_{n}$ - входные отсчеты во временной области, $k$ - выходные отсчеты в частотной области. 


\section{4. Определение эмоционального возбуждения}

Известным маркером когнитивных процессов являются перестройки ритмов мозга, проявляющиеся в поверхностно регистрируемой ЭЭГ человека. Сильное эмоциональное переживание, как форма когнитивной деятельности, способно привести к торможению других психических процессов, реализации поведенческих соответствующих реакций, нарушению сознательного контроля над действиями, в результате чего могут совершаться неконтролируемые поступки [7]. Состояния возникают помимо воли, не возможен сознательный контроль над своими действиями. Возникновение подобных ситуаций может привести к появлению критической ошибки в контуре управления. Поэтому в первую очередь была поставлена задача определения уровня эмоционального возбуждения человекаоператора.

\section{5. Результаты эксперимента}

В ходе исследования фиксировался сигнал ЭЭГ оператора при нахождении в спокойном состоянии и состоянии стресса. Исходный сигнал каждого датчика, с частотой дискретизации 128 Гц, разложен на частотные ритмы с помощью дискретного преобразования Фурье. Для визуальной оценки различий эмоциональных состояний построены графики спектральной мощности сигналов с датчиков $\mathrm{AF} 3, \mathrm{AF} 4, \mathrm{~F} 7, \mathrm{~F} 3, \mathrm{~F} 4, \mathrm{~F} 8, \mathrm{FC} 5, \mathrm{FC} 6, \mathrm{~T} 7, \mathrm{~T} 8, \mathrm{O} 1, \mathrm{O} 2$ в диапазоне значимых частот от 1 до 50 Гц. (см. Рис. 4, 5).

Проведен сравнительный анализ спектральной мощности различных эмоциональных состояний для лобной, височной, и затылочно-теменной долей коры головного мозга (ГМ) (см. Рис. $6,7,8)$.

На основе оценки общей тонической активности, а также значений суммарной спектральной мощности частотных диапазонов, сделан вывод о возможности оценки эмоционального фона человека-оператора. На Рис. 9 и 10 представлены графики интегрального накопления активации сигнала для различных эмоциональных состояний.

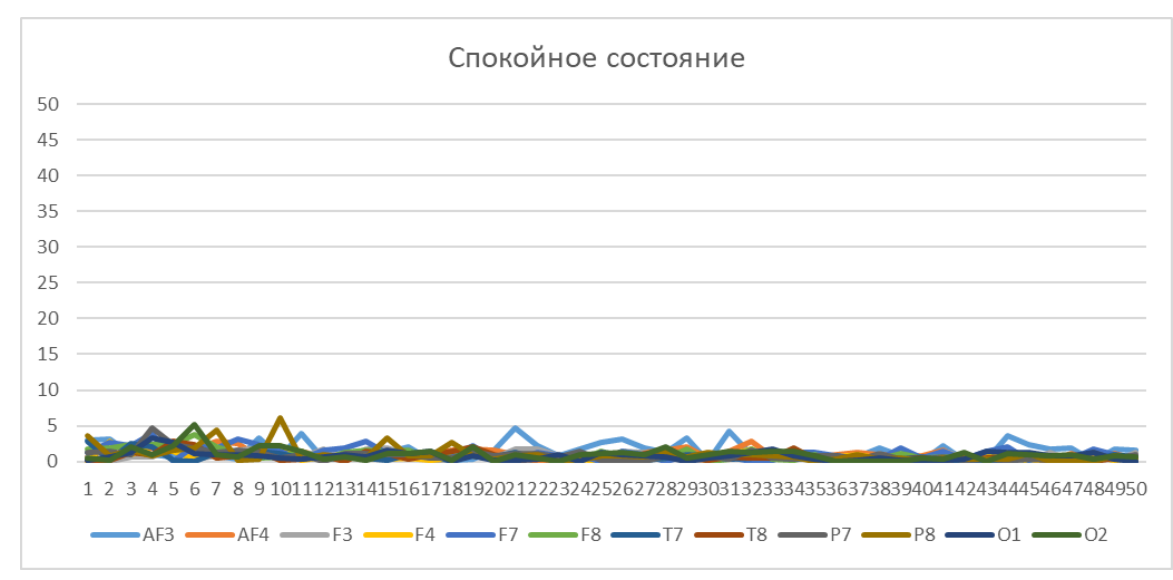

Pис. 4: График спектральной плотности мощности сигнала ЭЭГ, полученный дискретным преобразованием Фуръе для спокойного состояния 


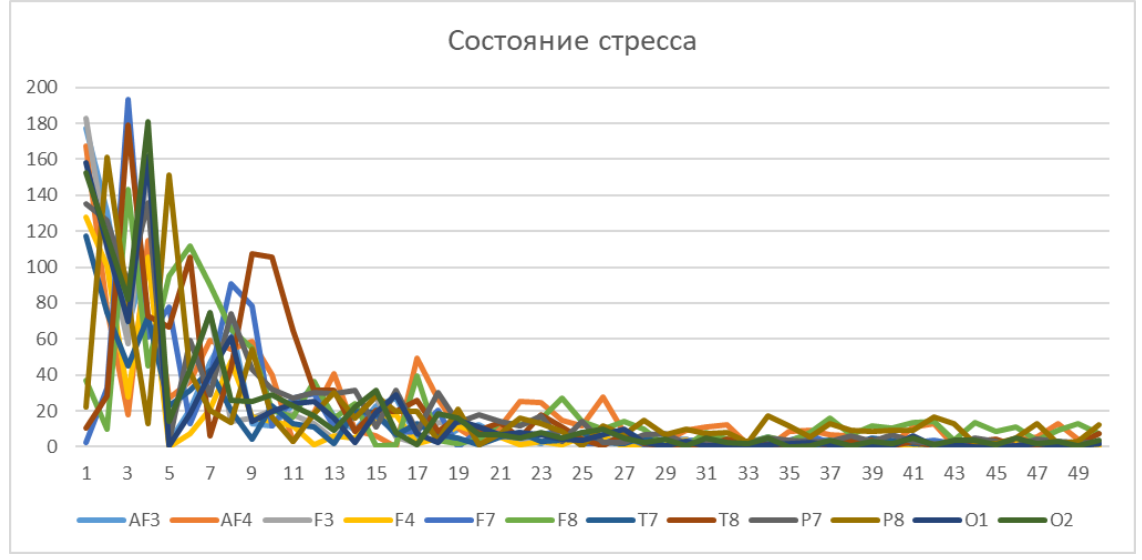

Рис. 5: График спектральной плотности мощности сигнала ЭЭГ, полученный дискретным преобразованием Фуръе для состояния стресса

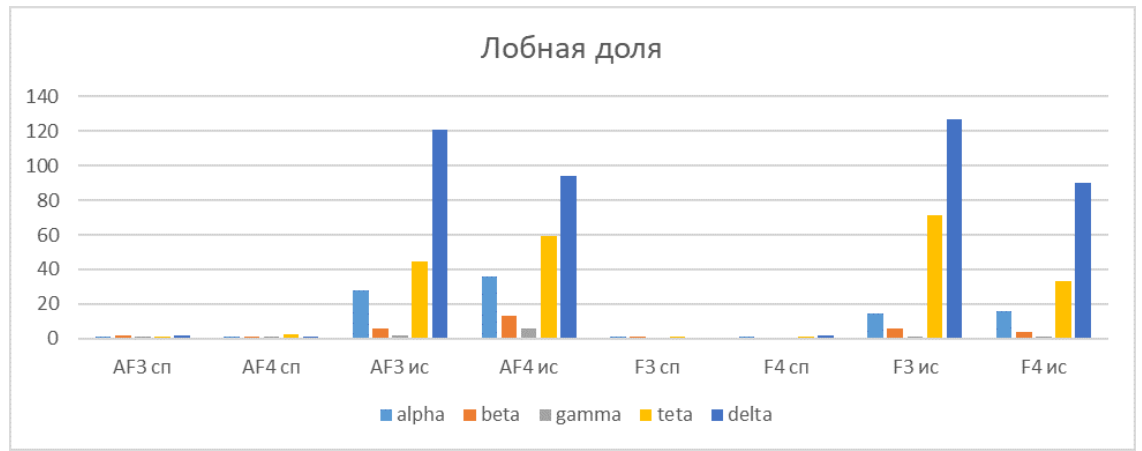

Рис. 6: Уровень спектральной мощности сигнала ЭЭГ для датчиков АFЗ, АF F3, F4 по каждой из частот по двум эмоциональным состояниям: сп - спокойное, ис-испуг

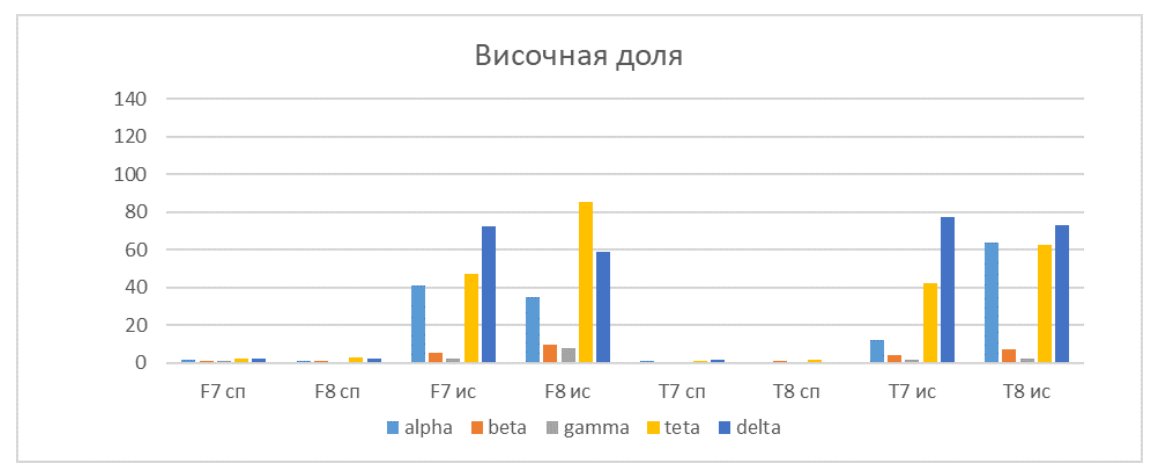

Рис. 7: Уровень спектральной мощности сигнала ЭЭГ для датчиков F7, F8, T7, Т8 по каждой из частот по двум эмоциональным состояниям: сп - спокойное, uc - ucny2 


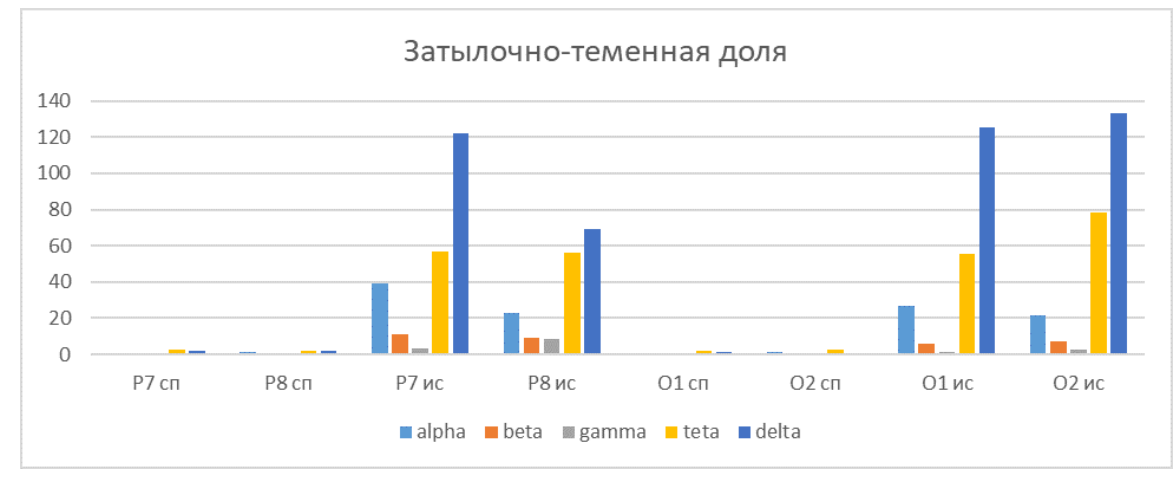

Рис. 8: Уровенъ спектральной мощности сигнала ЭЭГ для датчиков РҒ, Р8, О1, О2 по каждой из частот по двум эмоциональным состояниям: сп - спокойное, uc - ucnyz

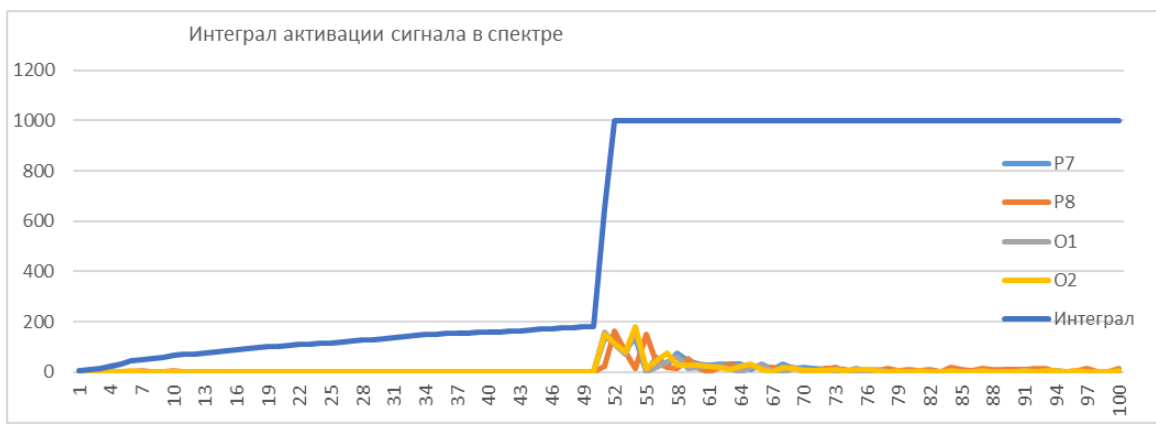

Рис. 9: Накопление активации сигнала при выставлении порогового значения в 1000 мкB

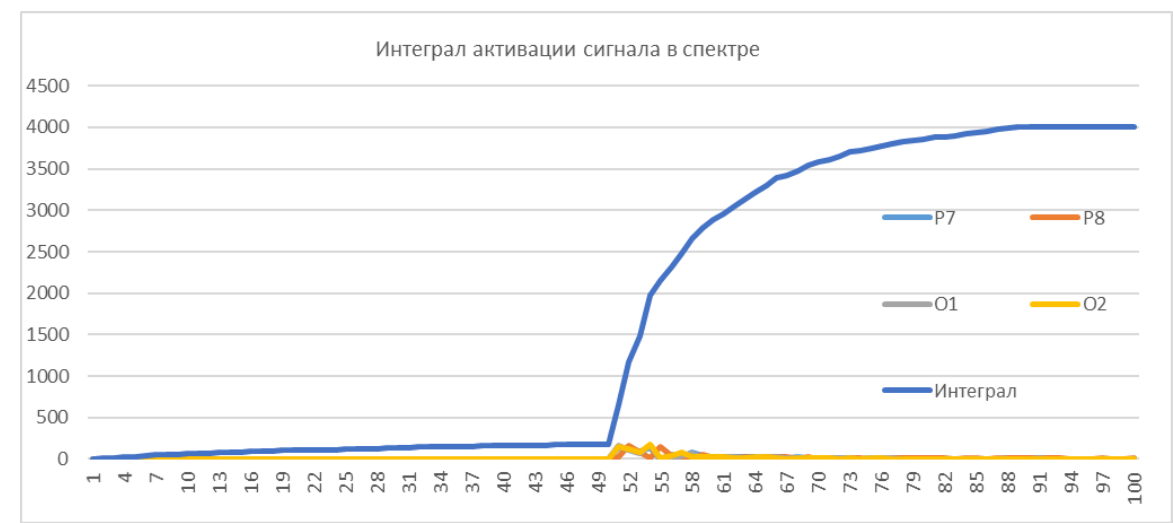

Рис. 10: Накопление активации сигнала при выставлении порогового значения в 4000 мк $B$

В спокойном состоянии интеграл сигнала не превышает отметку в 200 мкВ, в то время как в состоянии эмоционального возбуждения, пороговое значение 1000 мкВ достигается за одну итерацию, а 4000 мкВ - за 38 итераций. 
Регистрируемый программным продуктом сигнал ЭЭГ, используя БЗ, возвращает коэффициент, определяющий уровень эмоционального возбуждения. На основе этого коэффициента путем экспертной оценки на экран выводится предупреждение об уровне эмоционального возбуждения (см. Рис. 11).

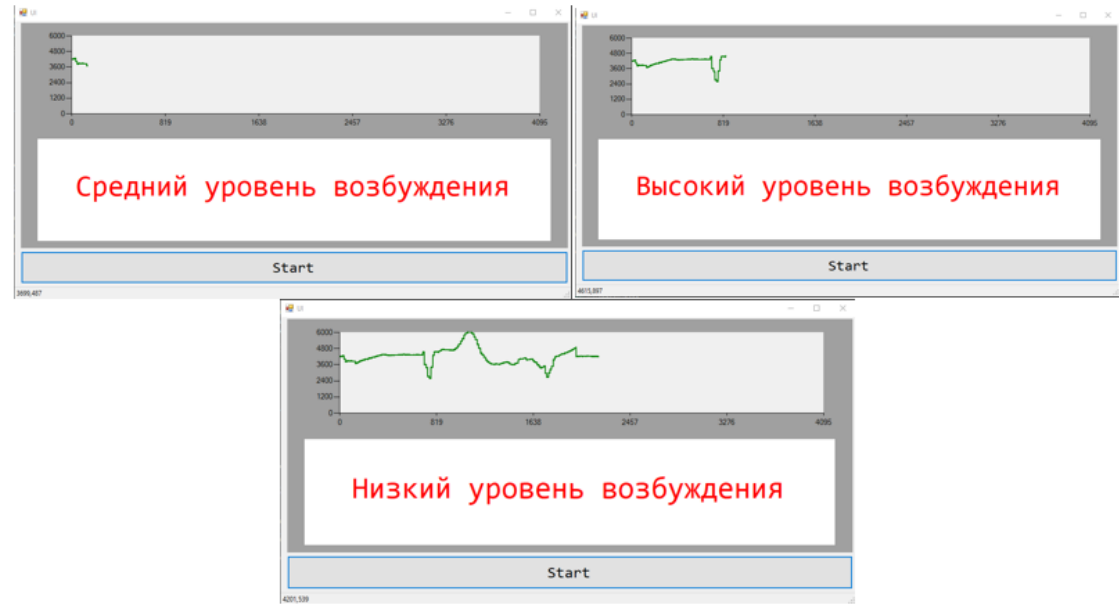

Рис. 11: Предупреждение об уровне эмоционального возбуждения

\section{6. Определение ментального состояния человека-оператора}

Эмоции, не зависимо от их знака, могут генерироваться в обоих полушариях ГМ, но при этом ряд исследований акцентирует внимание на причастности передних отделов ГМ к генерации эмоций (см. Рис. 12) [8].
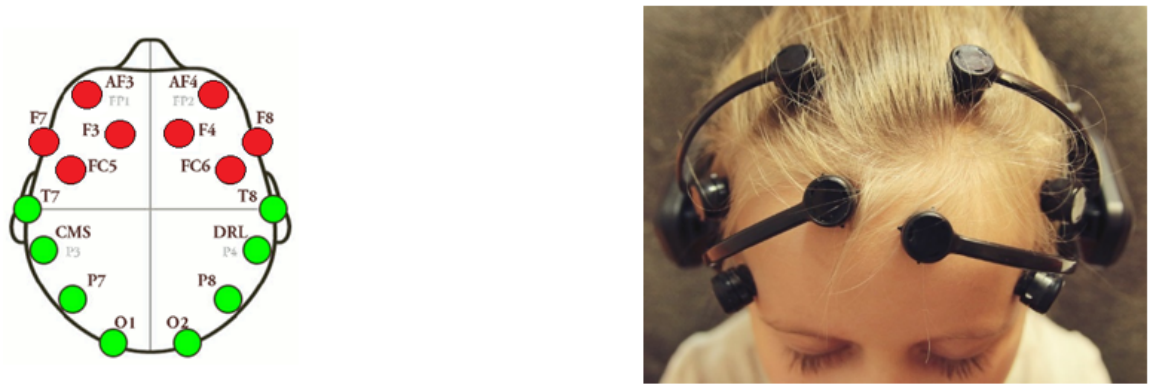

Рис. 12: Расположение электродов, исполъзуемых в ходе эксперимента

Совокупность одновременно присутствующих ритмов образует специфический пространственно-частотный паттерн ЭЭГ. Паттерны характерны для разных типов когнитивной деятельности и высоко индивидуально специфичны. Способность индивидуума к установлению ритмических паттернов ЭЭГ при выполнении определенных когнитивных заданий составляет «энцефалографический» портрет его личности [9]. Проведен эксперимент, в ходе которого записывался 
сигнал ЭЭГ с частотой дискретизации 128 Гц при различных эмоциональных состояниях: спокойном (нейтральном), положительных эмоциях и отрицательных эмоциях. Состояния сильного эмоционального возбуждения отмечено не было. Исходный сигнал каждого датчика, разложен на частотные ритмы с помощью дискретного преобразования Фурье. На Рис. 13 представлен график средней спектральной мощности диапазонов частот за 6 секунд для датчика AF3 при различных эмоциональных состояниях.

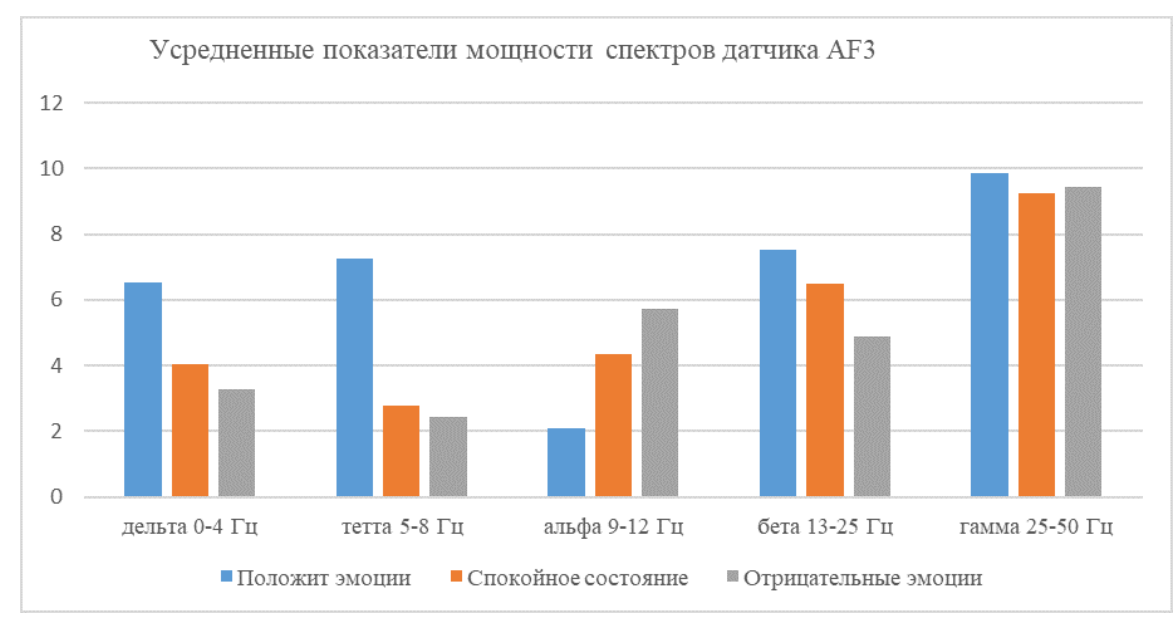

Рис. 13: Средние показатели мощности спектров датчика АFЗ

Определение знака эмоции - классическая задача классификации. Для ее решения необходимо определить знак эмоции в конкретный момент времени посредством анализа сигнала ЭЭГ. Если рассматривать спокойное состояние как 0 , положительную эмоцию как +1 , отрицательную как -1 , то определение знака эмоции можно рассматривать как отклонение от нейтрального состояния. Ряд исследований показывает [10], что при определении знака эмоциональной реакции необходимо опираться на изменения мощности альфа-ритма и бета-ритма в лобных и височных отведениях. При положительных эмоциях наблюдается сильная депрессия альфа-ритма в сравнении с нейтральным условием, а также увеличение мощности бета-ритма (см. Таблицу 1$)$.

В соответствии со значимостью спектральной мощности, расставлены коэффициенты, отражающие вес частотного ритма для определения знака эмоциональной активности.

\section{7. Описание программной платформы}

В качестве программной платформы для обработки сигнала ЭЭГ выбран язык программирования Python версии 3.7.1. В качестве библиотеки для математических операций был выбран пакет NumPy - это фундаментальный пакет для научных вычислений в Python, предоставляющий:

• мощный N-мерный объект массива;

- сложные (широковещательные) функции;

- инструменты для интеграции кода С / C ++ и Fortran; 
Таблица 1: Частотные ритмы при различных эмоциональных знаках

\begin{tabular}{|c|c|c|}
\hline Частотный ритм & $\begin{array}{l}\text { Положительные эмо- } \\
\text { ции }\end{array}$ & $\begin{array}{l}\text { Отрицательные эмо- } \\
\text { ции }\end{array}$ \\
\hline Альфа-ритм & $\begin{array}{l}\text { Более выраженная де- } \\
\text { прессия альфа-ритма в } \\
\text { сравнении с нейтраль- } \\
\text { ным условием в лобных } \\
\text { и височных отведениях }\end{array}$ & $\begin{array}{l}\text { Мощность альфа ритма } \\
\text { больше или равна мощ- } \\
\text { ности альфа-ритма при } \\
\text { нейтральном условии в } \\
\text { лобньх и височных отве- } \\
\text { дениях }\end{array}$ \\
\hline Бета-ритм & $\begin{array}{l}\text { Увеличение } \\
\text { бета-ритма в } \text { сравнентии } \\
\text { с нейтральным условием } \\
\text { в лобных и височных } \\
\text { отведениях }\end{array}$ & $\begin{array}{l}\text { Снижение амплитуды в } \\
\text { сравнении с нейтраль- } \\
\text { ным условием в лобных } \\
\text { отведениях }\end{array}$ \\
\hline Тета-ритм & $\begin{array}{l}\text { Изменение мощности } \\
\text { тета-ритма в лобных и } \\
\text { височньх отведениях } \\
\text { в сравнение с ней- } \\
\text { тральным условием в } \\
\text { зависимости от пола }\end{array}$ & $\begin{array}{l}\text { Изменение мощности } \\
\text { тета-ритма в лобных и } \\
\text { височных отведениях } \\
\text { в сравнение с ней- } \\
\text { тральным условием в } \\
\text { зависимости от пола }\end{array}$ \\
\hline
\end{tabular}

- алгоритмы линейной алгебры, преобразование Фурье и расширенные возможности для генерации случайных чисел.

Помимо очевидных научных применений, NumPy можно также использовать в качестве эффективного многомерного контейнера общих данных. Возможность определения произвольных типов данных позволяет легко и быстро интегрироваться с широким спектром баз данных.

\section{8. Применение глубокого машинного обучения в задаче классификации эмоционального знака}

В качестве библиотеки для машинного обучения использовалась TensorFlow это нейронная сеть, которая в процессе обработки данных обучается решать задачи путем позитивного усиления и обрабатывает данные на различных уровнях (узлах), что помогает находить искомый верный результат с заданным уровнем ошибки аппроксимации обучающего сигнала. Машинное обучение такого рода очень хорошо адаптировано для исследовательских целей.

Библиотека была разработана командой Google Brain для множества задач, включая поиск изображений и улучшение алгоритмов распознавания речи. В качестве отправной точки применения технологий машинного обучения (а далее - мягких вычислений), для распознавания эмоций через сигнал ЭЭГ, библиотека TensorFlow заметно упрощают встраивание в приложения самообучаемых элементов и функций искусственного интеллекта, предназначенных для распознавания речи, организации компьютерного зрения или обработки естественного языка. 
Принцип работы с TensorFlow подразумевает составление графа операций, передачу данных и произведение вычислений. Графы позволяют определить процесс вычисления, где вершины выполняют операции, а ребра описывают связь между ними. Таким образом, при сложении двух чисел, необходимо определить вершину с двумя входами (числа для сложения), некоторые вычисления (функция сложения двух чисел) и выход (результат).

Цель применения технологии машинного обучения - определить знак эмоции. Чтобы TensorFlow мог обучать модель, необходимо задать функцию потерь. В обучающий алгоритм на вход подаются значения мощности спектров по каждой из частот (альфа, бета, гамма, тета, дельта). Задача распознавания эмоции является задачей классификации, поэтому функция потерь будет возвращать:

- При нейтральном состоянии - 0.

- При отрицательном состоянии - 1 .

- При положительном состоянии -+1 .

На Рис. 14 и 15 представлена визуализация данных, подаваемых на вход нейронной сети. Рис. 14 отражает общую тоническую активность спектров эмоций разного знака для датчиков, находящихся в одном полушарии ГМ, а Рис. 15 датчиков, симметрично располагающихся на противоположных точках двух полушарий ГМ.

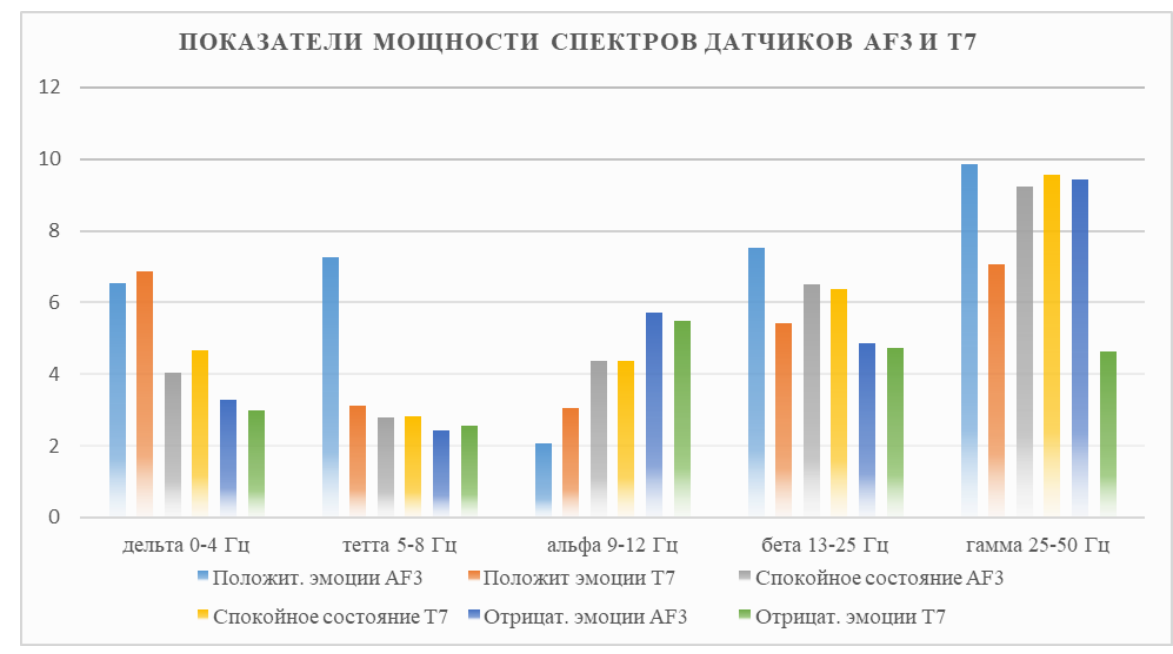

Рис. 14: Показатели мощности спектров датчиков АFЗ и Т7 для положительных, отрицательных эмоций и спокойного состояния

\section{9. Оптимизатор баз знаний}

На Рис. 16 показан результат работы нейронной сети: коэффициент отклонения от нейтрального состояния, полученный после обработки сигнала ЭЭГ, разложенного на частотные диапазоны. 


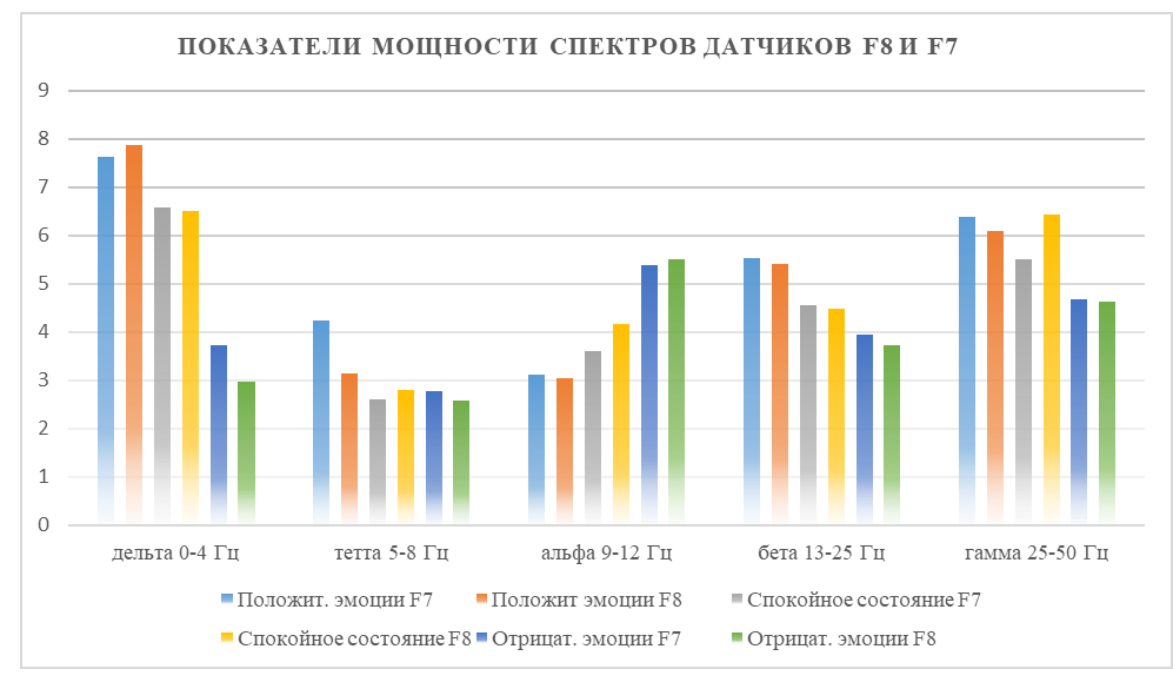

Рис. 15: Показатели мощности спектров датчиков F7 и F8 для положительных, отрицательных эмоций и спокойного состояния

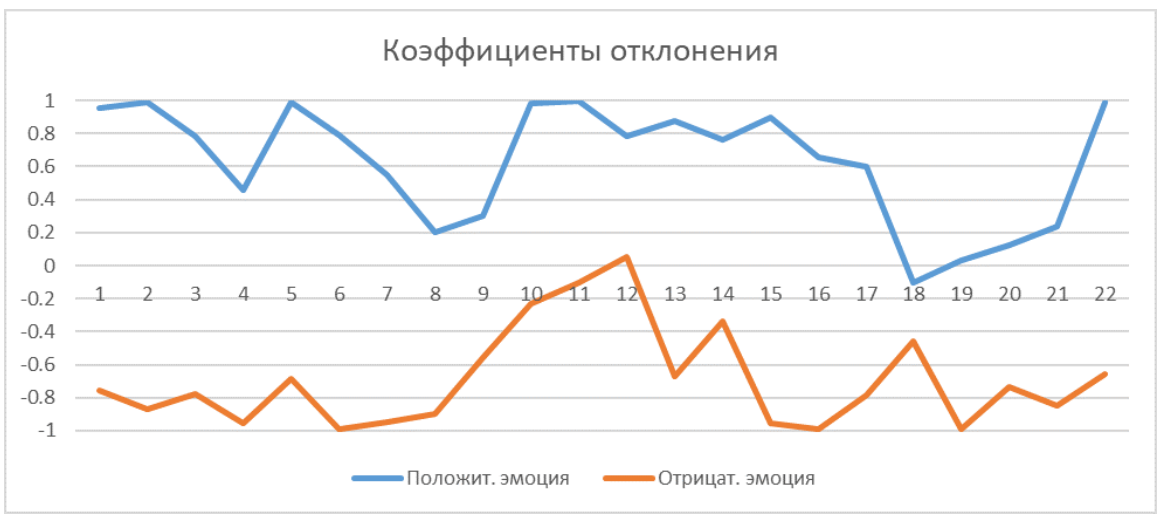

Рис. 16: Коэффициенты отклонения от нейтрального состояния эмоций разного знака

В рамках решения поставленной задачи, машинное обучение используется для определения знака эмоции в определенный момент времени. Для корректного описания общего психофизического состояния оператора необходимо применение оптимизатора баз знаний на мягких вычислениях. Поскольку эмоции характеризуются отчетливо выраженной интенсивностью, ограниченной продолжительностью, осознанностью причины ее появления; связью с конкретным объектом, обстоятельством; полярностью, необходима аппроксимация коэффициента отклонения от нейтрального состояния.

Для создания базы знаний на мягких вычислениях используется ПО SCOptimizer. Оптимизатор баз знаний на основе мягких вычислений (Оптимизатор баз знаний, ОБ3, Soft Computing Optimizer, SCOptimizer ${ }^{\mathrm{TM}}$ ) является программным средством для создания автоматических нечетких моделей. Оптимизатор баз знаний использует наборы значений вектора вход-выход для создания 


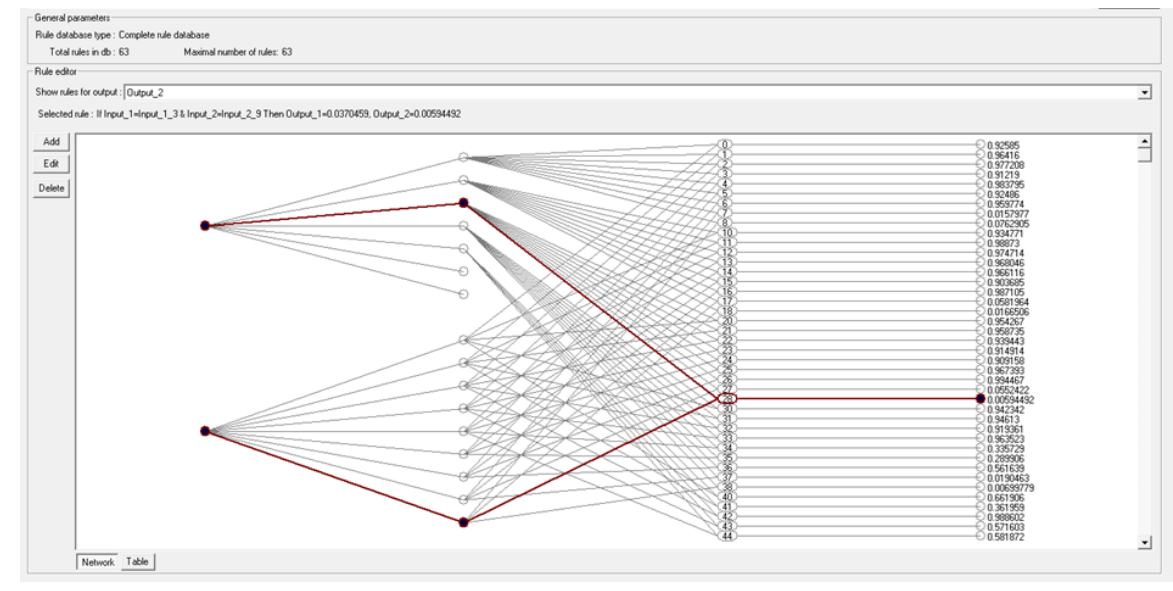

Рис. 17: База правил в виде сети с четыръмя слоями

и оптимизации нечеткой модели. Для выполнения различных алгоритмов оптимизации необходим обучающий сигнал, который представляет образцы входных значений и соответствующих выходных значений. Файлы обучающего сигнала могут быть созданы с помощью Оптимизатора баз знаний или взяты из других источников. Текстовые файлы обрабатываются на основе региональных настроек, которые определяют символы для десятичной точки, разделителя тысяч и так далее. По умолчанию используются значения этих параметров, установленные в Windows. Если настройки не соответствуют формату сигнала, то они могут быть изменены в любой момент. После изменения параметры сохраняются в модели и будут использоваться для дальнейшей обработки данных. Региональные настройки влияют на чтение и запись текстовых данных и файлов модели.

SCOptimizer $^{\mathrm{TM}}$ поддерживает экспорт модели в программе на языке С. Код в этих файлах написан с минимальным использованием функций из стандартной библиотеки языка $\mathrm{C}$ и может быть скомпилирован любым компилятором $\mathrm{C}$, включая ориентированные на встраиваемые системы и микроконтроллеры [5]. Для аппроксимации обучающего сигнала применяется оптимизатор баз знаний с выбранной моделью нечеткого вывода (Сугено 0 порядка). Используется коэффициент отклонения от нейтрального состояния и идентификационное значение соответствующей эмоции. На следующем этапе проектирования базы знаний для нечеткого вывода осуществляется формирование в автоматическом режиме полной базы знаний, и дальнейшая оптимизация правых частей правил (см. Рис. 17).

Первый слой - слой входных переменных: спектральная плотность мощности сигнала и экспертная оценка. Второй слой - нечёткие терм-множества входных переменных. Третий слой соответствует правилам базы знаний с соответствующим номером правила в базе правил. Последний слой - выходной слой, который отображает числовые параметры правила. Оптимизатор баз знаний на мягких вычислениях автоматически формирует оптимальную структуру нейронной сети, позволяет аппроксимировать обучающий сигнал с требуемой (заданной) ошибкой аппроксимации, реализует алгоритм глубокого машинного обучения [11]. На Рис. 17 , в первом графике изображен обучающий сигнал и результат модели выходной переменной.

Линия зеленого цвета отображает обучающий сигнал, линия синего цвета - 


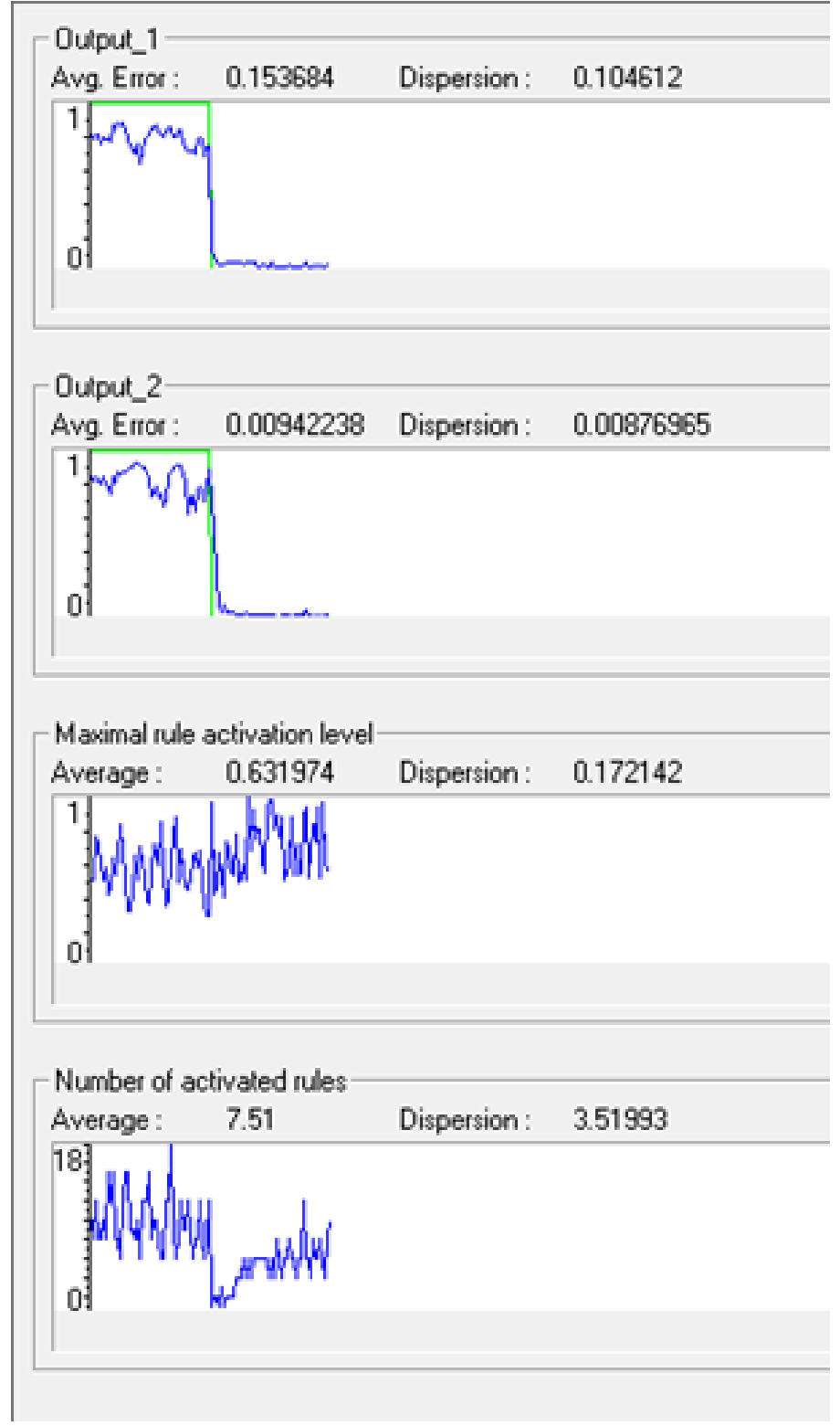

Рис. 18: Результат модели выходной переменной

выход модели. На втором и третьем графиках максимальный уровень активаций правил и количество активированных правил [12].

\section{Заключение}

Проведенная работа показала принципиальную возможность классификации ментальных состояний человека-оператора, демонстрирует оптимальную обучаемость системы, возможность создания баз знаний на основе регистрируемого сигнала ЭЭГ и использования полученных результатов для распознавания 
эмоций. Поскольку эмоции характеризуются отчетливо выраженной интенсивностью, ограниченной продолжительностью, осознанностью причины ее появления, связью с конкретным объектом, обстоятельством и полярностью, то использование машинного обучения и интеллектуальной надстройки в виде ОБЗ, основанной на нечеткой логике, является оптимальным инструментом для корректного описания общего психофизического состояния оператора. Робастность ИСУ, полученная на основе такого подхода, требует минимума исходной информации как о поведении объекта управления, так и о внешних возмущениях.

\section{Список литературы}

[1] Петров Б.Н., Уланов Г.М., Ульянов С.В., Хазен Э.М. Информационносемантические проблемы в процессах управления и организации. М.: Наука, 1977. 452 c.

[2] Noor A.K. Potential of cognitive computing and cognitive systems // Open Engineering. 2015. Vol. 5, № 1. Pp. 75-88. https://doi.org/10.1515/eng-20150008

[3] Hieida C., Horii T., Nagai T. Deep Emotion: A Computational Model of Emotion Using Deep Neural Networks [Electronic resource] // Computing Research Repository. 2018. URL: http://arxiv.org/abs/1808.08447.

[4] Розалиев В.Л. Построение математической модели эмоций // Сборник научных трудов $\mathrm{V}$ международной научно-практической конференции «Интегрированные модели и мягкие вычисления в искусственном интеллекте» (Коломна, 28-30 мая 2009 г.). Т. 2. М.: Физматлит. С. 950-957.

[5] Ульянов С.В., Литвинцева Л.В., Добрынин В.Н., Мишин А.А. Интеллектуальное робастное управление: технологии мягких вычислений. М.: ВНИИгеосистем, 2011. 406 с.

[6] Ульянов C.В., Решетников А.Г., Мамаева А.А., Скотников С.В. Гибридные когнитивные системы управления на примере управления транспортным средством // Системный анализ в науке и образовании. 2016. № 2. URL: http:/www.sanse.ru/archive/40

[7] Fretska E., Bauer H.U., Leodolter M., Leodolter U. Loss of control and negative emotions: a cortical slow potential topography study // International Journal of Psychophysiology. 1999. № 33. Pp. 127-141.

[8] Николаев А.Р. Спектральные характеристики ЭЭГ на первом этапе решения различных пространственных задач / / Психологический журнал. 1994. Т. 15, № 6. C. 100-106.

[9] Ульянов С.В., Мамаева А.А., Шевченко А.В. Когнитивно-интеллектуальная система диагностики, обучения и адаптации детей-аутистов // Системный анализ в науке и образовании. 2016. № 5. URL: http:/www.sanse.ru/archive/42

[10] Лапшина Т.Н. Психофизиологическая диагностика эмоций человека по показателям ЭЭГ // Материалы Международной научно-практической конференции «Развитие научного наследия Бориса Михайловича Теплова в отечественной и мировой науке» (Москва, 15-16 ноября 2006 г.). М.: БФ «Твердислов». С. 160-165. 
[11] Ulyanov S.V. Soft computing optimizer of intelligent control system structures. US Patent No 7,219,087B2. Date of patent: May 15, 2007 [WO 2005/013019 A3, 2005].

[12] Ульянов С.В., Решетников А.Г., Мамаева А.А. Гибридные когнитивные нечеткие системы управления автономным роботом на основе нейроинтерфейса и технологии мягких вычислений // Программные продукты и системы. 2017. Т. 30, № 3. С. 420-424. http://dx.doi.org/10.15827/0236235X.119.420-424

\section{Образец цитирования}

Ульянов С.В., Мамаева А.А., Шевченко А.В. Технология когнитивного интеллектуального управления: оптимизатор баз знаний на мягких вычислениях и глубокое машинное обучение // Нечеткие системы и мягкие вычисления. 2018. T. 13, № 2. C. 166-182. https://doi.org/10.26456/fssc44

\section{Сведения об авторах}

\section{1. Ульянов Сергей Викторович}

профессор кафедры системного анализа и управления государственного университета «Дубна».

Россия, 141982, Московская обл., г. Дубна, ул. Университетскал, д. 19.

E-mail: ulyanovsv@mail.ru

2. Мамаева Алла Александровна

аспирант кафедры системного анализа и управления государственного университета «Дубна».

Россия, 141982, Московская обл., г. Дубна, ул. Университетскал, д. 19.

E-mail: allamamaeva.d@gmail.com

\section{3. Шевченко Андрей Владимирович}

аспирант кафедры системного анализа и управления государственного университета «Дубна».

Россия, 141982, Московскал обл., г. Дубна, ул. Университетскал, д. 19. 


\title{
THE COGNITIVE TECHNOLOGY OF INTELLIGENT CONTROL: SOFT COMPUTING OPTIMIZER AND DEEP MACHINE LEARNING
}

\author{
Ulyanov Sergey Viktorovich
}

Professor at System Analysis and Management department, Dubna State University Russia, 141982, Moscow region, Dubna, Universitetskaya str., 19.

E-mail: ulyanovsv@mail.ru

Mamaeva Alla Aleksandrovna

Postgraduate student of System Analysis and Management department, Dubna State University

Russia, 141982, Moscow region, Dubna, Universitetskaya str., 19.

E-mail: allamamaeva.d@gmail.com

Shevchenko Andrey Vladimirovich

Postgraduate student of System Analysis and Management department, Dubna State University

Russia, 141982, Moscow region, Dubna, Universitetskaya str., 19.

Received 05.12.2018, revised 17.12.2018.

The article discusses the possibility of using the deep machine learning technology based on the soft computing optimizer in cognitive intelligent control using a cognitive helmet as a neural interface. The goal of this work is to experimentally show the possibility of classifying the mental states of a human operator, to identify objective indicators of the psychophysiological state of the examined person. The role and necessity of applying intelligent calculations in the task of describing the general psychophysical state of an operator is shown with examples.

Keywords: neural interface, intelligent computing, intelligent control system, deep machine learning, emotions.

\section{Citation}

Ulyanov S.V., Mamaeva A.A., Shevchenko A.V., "The cognitive technology of intelligent control: soft computing optimizer and deep machine learning", Nechetkie Sistemy i Myagkie Vychisleniya [Fuzzy Systems and Soft Computing], 13:2 (2018), 166-182 (in Russian). https://doi.org/10.26456/fssc44

\section{References}

[1] Petrov B.N., Ulanov G.M., Ulyanov S.V., Khazen E.M., Informatsionnosemanticheskie problemy $v$ protsessakh upravleniya $i$ organizatsii [Information and semantic problems in management and organization processes], Nauka Publ., Moscow, 1977 (in Russian), 452 pp.

[2] Noor A.K., "Potential of cognitive computing and cognitive systems", Open Engineering, 5:1 (2015), 75-88, https://doi.org/10.1515/eng-2015-0008. 
[3] Hieida C., Horii T., Nagai T., Deep Emotion: A Computational Model of Emotion Using Deep Neural Networks, Computing Research Repository, 2018, http://arxiv.org/abs/1808.08447.

[4] Rozaliev V.L., "Construction of a mathematical model of emotions", Sbornik nauchnykh trudov $V$ mezhdunarodnoj nauchno-prakticheskoj konferentsii "Integrirovannye modeli i myagkie vychisleniya $v$ iskusstvennom intellekte" [Proceedings of the $V$ international scientific-practical conference "Integrated models and soft computing in artificial intelligence"]. V.2 (Kolomna, 28-30 maya 2009 g.), Fizmatlit Publ., Moscow, 950-957 (in Russian).

[5] Ulyanov S.V., Litvintseva L.V., Dobrynin V.N., Mishin A.A., Intellektualnoe robastnoe upravlenie: tekhnologii myagkikh vychislenij [Intelligent Robust Control: Soft Computing Technologies], VNIIgeosistem Publ., Moscow, 2011 (in Russian), 406 pp.

[6] Ulyanov S.V., Reshetnikov A.G., Mamaeva A.A., Skotnikov S.V., "Hybrid cognitive control systems on the example of driving", Sistemnyj analiz $v$ nauke $i$ obrazovanii [System analysis in science and education], 2016, № 2 (in Russian), http:/www.sanse.ru/archive/40.

[7] Fretska E., Bauer H.U., Leodolter M., Leodolter U., "Loss of control and negative emotions: a cortical slow potential topography study", International Journal of Psychophysiology, 1999, № 33, 127-141.

[8] Nikolaev A.R., "Spectral characteristics of the EEG at the first stage of solving various spatial problems", Psikhologicheskij zhurnal [Psychological Journal], 15:6 (1994), 100-106 (in Russian).

[9] Ulyanov S.V., Mamaeva A.A., Shevchenko A.V., "Cognitive-intellectual system of diagnostics, training and adaptation of children with autism", Sistemnyj analiz $v$ nauke $i$ obrazovanii [System analysis in science and education], 2016, № 5 (in Russian), http:/www.sanse.ru/archive/42.

[10] Lapshina T.N., "Psychophysiological diagnosis of human emotions in terms of EEG", Materialy Mezhdunarodnoj nauchno-prakticheskoj konferentsii "Razvitie nauchnogo naslediya Borisa Mikhajlovicha Teplova $v$ otechestvennoj $i$ mirovoj nauke" [Materials of the International Scientific and Practical Conference "Development of the Scientific Heritage of Boris Mikhailovich Teplov in the Russian and World Science"] (Moscow, November 15-16, 2006), BF «Tverdislov», Moscow, 160-165 (in Russian).

[11] Ulyanov S.V., Soft computing optimizer of intelligent control system structures, US Patent No 7,219,087B2. Date of patent: May 15, 2007 [WO 2005/013019 A3, 2005].

[12] Ulyanov S.V., Reshetnikov A.G., Mamaeva A.A., "Hybrid cognitive fuzzy control systems for an autonomous robot based on neurointerface and soft computing", Programmnye Produkty i Sistemy [Software and Systems], 30:3 (2017), 420-424 (in Russian), http://dx.doi.org/10.15827/0236-235X.119.420-424. 